A collaboration between the JAOA and the American Association of Colleges of Osteopathic Medicine (AACOM) to recruit, peer review, publish, and distribute research and other scholarly articles related to osteopathic medical education.

\title{
JAOA/AACOM
}

\section{Interprofessional Education: Collaboration and Learning in Action}

Stephanie H. Felgoise, PhD; Jeffrey Branch, EdD; Ashley Poole, MA, MS; Laura Levy, DHSc, PA-C; Michael Becker, DO, MS

From the Philadelphia College of Osteopathic Medicine in Pennsylvania.

Financial Disclosures: None reported.

Support: None reported.

Address correspondence to Stephanie H. Felgoise, PhD,

School of Professional \& Applied Psychology,

Department of Clinical Psychology, 4190 City Ave, Philadelphia, PA 19131-1610.

Email: stephanief@pcom.edu

Submitted October 9, 2018; revision received March 10, 2019; accepted April 23, 2019.
Interprofessional education (IPE) is necessary to ensure that future health care professionals are prepared to provide holistic, patient-centered prevention programs, assessments, diagnoses, treatment plans, and chronic illness management in a collaborative manner. Accrediting bodies such as the Commission on Osteopathic College Accreditation and the American Psychological Association newly require programs to implement instruction and evaluate IPE core competency development in each year of their programs. The IPE core team at the Philadelphia College of Osteopathic Medicine has designed, implemented, and tested an IPE core course over the past 2 years that includes students in osteopathic medicine, clinical psychology, mental health counseling, and physician assistant programs. Throughout this process, the IPE core team has identified strengths, weaknesses, opportunities, and challenges. Cultural considerations, institutional resources, pedagogy for large interdisciplinary groups at different stages of training, and technology and assessment tools for student and course evaluation are all critical considerations.

J Am Osteopath Assoc. 2019;119(9):612-619

doi:10.7556/jaoa.2019.109

Keywords: core competancy, interprofessional education, interprofessional practice, patient care

$\mathrm{H}$ ealth care education programs are challenged to expand biopsychosocial spiritual curricula and training of health care professionals from independent disciplines to integrative and collaborative teams. ${ }^{1,2}$ The philosophy is that students across disciplines need to learn "about, from and with each other to enable effective collaboration and improve health outcomes.",2,3 Professionals in various disciplines cannot be expected to work together as a health care team if they did not train with students and faculty of different disciplines. ${ }^{2}$

Fourteen organizations, including the American Association of Colleges of Osteopathic Medicine, the American Psychological Association, and the Physician Assistant Education Association, implemented training requirements that include interprofessional and collaborative practice principles and experiences in accordance with the Interprofessional Education Collaborative Practice (IPEC) initiative. ${ }^{4}$ In $2016,{ }^{5}$ IPEC established core 
competencies for health care professionals to obtain, and these competencies are the foundation for the goals and objectives of the interprofessional education (IPE) initiative at Philadelphia College of Osteopathic Medicine. The IPEC competencies include: values/ethics, roles/ responsibilities, teams/teamwork, and professional communication. ${ }^{4,5}$ The World Health Organization has observed the association between IPE, collaborative care, and improved patient outcomes. ${ }^{2}$ Studies support the positive impact IPE can have on student attitudes, collaborative skills, and patient outcomes. ${ }^{6-8}$ Medical students engaging in sessions that emphasize interprofessional collaboration have an opportunity to enhance their understanding of different professional roles, improve their ability to communicate with health care professionals, and develop their ability to work on a team. The IPE course at PCOM was built on the belief that these skills are foundational for better patient care.

Few examples exist to guide institutions on establishing IPE curricula. ${ }^{9,10-14}$ Leadership and coordination of IPE efforts vary across institutions. Likewise, represented disciplines are dependent on university offerings. Although the PCOM doctor of osteopathic medicine (DO) course curriculum includes IPE in all 4 years of education and training, the IPE core team created a course specifically for fourth-year DO students, second-year doctor of psychology (PsyD) students, second-year master of science in mental health counseling (MHC) students, and first-year physician assistant (PA) students. The purpose of this article is to describe this course and to present the strengths, weaknesses, opportunities, and challenges we identified that may also benefit other programs in the development stages of their IPE curriculum.

\section{Course Participants}

\section{Students}

A total of 310 students participated (130 per session): 30 DO; 26 PsyD; 19 MHC; and 55 PA. Attendance was required as part of the DO students' ambulatory sub-internship rotation; PsyD students' integrative health care course; MHC students' professional development seminar; and PA students' interprofessional education course. As such, students may have had additional program-specific requirements to those assigned from the IPE course.

\section{Facilitators}

Interprofessional education leaders included the assistant dean of clerkship education, the director of the PsyD program in clinical psychology, the director of the program in organizational development and leadership, a PsyD candidate, and the chair of PA studies. Additional facilitators included 7 paid PsyD candidates, 2 psychology and 3 PA faculty members, and 6 faculty family medicine physicians.

\section{Additional Support}

The college president, the provost's administrative assistant, clinical psychology faculty, registrar personnel, the chief library services officer, the library associate director, the academic coordinator of professional development, and an academic coordinator in the online learning department served critical roles in delivery of the course.

\section{Course Description}

The course consisted of seven 2.5-hour sessions comprising 75 minutes of didactics with all students and 75 minutes of small-group sessions. Each session had its own Blackboard website. This site was the nucleus for posting announcements, course materials, tests, and survey links. Reading materials represented content on the session topic from journals reflecting each of the disciplines, and video links provided patient examples. Students completed 5-item multiple-choice pretests and posttests that were mobile-optimized and autoscored by Blackboard, with immediate learner feedback provided. Tests provided accountability for attendance, content learning, and course evaluation. Pre- and posttest results were not evaluated for this article. Students self-assigned to their small-group membership via Google Sheets links that were color-coded by 
Table 1.

The Tenets of Osteopathic Medicine Addressed in Each IPE Session

\begin{tabular}{llllll} 
& & $\begin{array}{c}\text { Tenets of } \\
\text { Osteopathic } \\
\text { Mesicine }\end{array}$ \\
\cline { 2 - 6 } Session & $\mathbf{1}$ & $\mathbf{2}$ & $\mathbf{3}$ & $\mathbf{4}$ \\
\hline Impairment & $\mathrm{X}$ & $\mathrm{X}$ & $\mathrm{X}$ & $\mathrm{X}$ \\
\hline Self-care & $\mathrm{X}$ & $\mathrm{X}$ & $\mathrm{X}$ & $\mathrm{X}$ \\
\hline Elder abuse & $\mathrm{X}$ & & $\mathrm{X}$ & $\mathrm{X}$ \\
\hline Care of patients with intellectual disabilities & $\mathrm{X}$ & $\mathrm{X}$ & & $\mathrm{X}$ \\
\hline Business of health care & & & & $\mathrm{X}$ & \\
\hline When Cultures Meet & & & & & $\mathrm{X}$ \\
\hline Care of patients with physical disabilities & $\mathrm{X}$ & $\mathrm{X}$ & $\mathrm{X}$ & $\mathrm{X}$
\end{tabular}

a (1) The body is a unit; the person is a unit of body, mind, and spirit. (2) The body is capable of self-regulation, self-healing, and health maintenance. (3) Structure and function are reciprocally interrelated. (4) Rational treatment is based upon an understanding of the above 3 principles.

Abbreviation: IPE, interprofessional education.

discipline with slots divided across the number of interprofessional small groups. Pairs comprising a medical faculty member and a psychology student or faculty member led each of the small-group sections.

Seven sessions included in the 2017-2018 curriculum focused on contemporary issues important to all disciplines and consistent with the tenets of osteopathic medicine (Table 1). ${ }^{15}$ Sessions 1 and 2 addressed psychological impairment of health care professionals and self-care, respectively. Lectures by DO and PsyD faculty members on these topics preceded small-group activities that engaged students in team role-playing with "patients" who represented persons with burnout, a substance use disorder, or a different psychiatric disorder. The teams created and executed biopsychosocial assessments and recommendations for treatment. Facilitators provided feedback and socratically guided problem-solving toward patients' goals. A “walk and talk," a facilitated walk around campus in interprofessional teams, required discussion about each other's disciplines and the strengths and challenges of their graduate studies and profession with regard to self-care and burnout. Facilitators and students agreed to share and commit to 1 new self-care activity in the upcoming week. Collaborative problem-solving and discussion regarding graduate students' barriers to self-care and the need for intentional efforts were addressed.

Session 3 was led by 2 physicians specializing in geriatrics and addressed how to assess for and respond to elder abuse. This topic was chosen because it is not consistently addressed across professions. Small-group exercises required IPE teams to role-play assessments of patient-caregiver dyads, with a focus on IPE skills and management of high-risk situations from a teambased approach.

The care of persons with intellectual disabilities was the focus of session 4. An administrator of an adult day services program presented patient-care and caregiver challenges for this population. Students engaged in questions and answers regarding IPE team care. In small-group activities, they learned about assessment, diagnosis, sexual health, behavioral factors relating to medical adherence, and health care professionals' roles in the care of adults with intellectual disabilities. Thus, learning about, from, and with other professionals regarding challenges and rewards of working with persons with intellectual disabilities provided a rich IPE experience.

The PCOM president taught students about the business of health care in session 5 , with an emphasis on social determinants of health. ${ }^{20}$ His lecture provided context for PCOM's commitment to serving underserved populations in its health care centers and the challenges holistic care and medicine face. Students were charged with the task of developing a hypothetical integrative health care practice in West Philadelphia and determining its budget. They were required to hire 2 physicians, 2 PAs, 1 MHC, 1 psychologist, and staff. Each interprofessional small group was assigned to develop one of the following aspects of the practice: (a) practice management software; (b) add-on services with existing staff; (c) facility costs; (d) staffing needs; (e) payer mix; (f) marketing; and (g) outcomes. Students populated their small groups' work in a 
shared Google Doc that was then projected to the whole class and presented to an interprofessional expert panel who asked questions pertinent to the IPEC competencies.

Session 6 was facilitated by the online learning department and PsyD program faculty using a copyrighted simulation, "When Cultures Meet." ${ }^{16}$ Culture was experienced in small groups, with in-group and out-group dynamics created for students to gain perspective of various constituents in different cultures. The simulation encouraged students to think of culture in the traditional sense of persons' identification with their own and others' characteristics and also to use this experience as a context for discussion about the culture of health care. The experiential design highlighted the interdependence of the IPEC core competencies. The session had didactic components, experiential learning, and debriefing, highlighting the impact of simulations as an opportunity for intentional and deeper immersion in the learning experience. ${ }^{17-19}$

Session 7 highlighted the care of persons with physical disabilities and was taught by a patient with cerebral palsy. Questions and answers were facilitated by his osteopathic physician. As part of students' pretest, they submitted questions for the patient so that he could program his responses in his augmentative speech device in advance. The emphasis of this class was on quality of life, individual differences, and comprehensive biopsychosocial care of patients using a team-based interprofessional and collaborative health care approach. Small-group activities included viewing a TED Talk depicting a female patient with cerebral palsy discussing her strengths and challenges, and participating in an interprofessional discussion about the health care and interprofessional competencies necessary to optimally provide services for this population.

\section{Survey and Outcomes}

Students completed anonymous surveys after each session, reporting their perceived utility of the content, format, and facilitators to advance their IPE; under- standing of the patient-professional relationship and their roles in relation to other health care professionals; knowledge about other professions; and recommendations for each module to be included in future IPE courses. A 4-point scale (not at all valuable, not very valuable, somewhat valuable, very valuable) rating did not provide a neutral response option.

Of approximately 130 students per session, the number of respondents to each satisfaction survey varied: session $1, \mathrm{n}=79$; session $2, \mathrm{n}=72$; session 3 , $\mathrm{n}=81$; session $4, \mathrm{n}=84$; session $5, \mathrm{n}=84$; session 6 , $\mathrm{n}=76$; session $7, \mathrm{n}=28$. Detailed information is presented in Table 2.

Small-group activities had high-value ratings for most class sessions by the majority of students, and the majority of students valued the IPE lectures.

A goal of IPE is to encourage students to think about their professional role in relation to other health care professionals. Our survey results suggest that students valued small-group activities and lectures in this regard. For example, to $82.3 \%(65 / 79)$ of students in session 1 and $64.5 \%(49 / 76)$ of students in session 6 found value in the small-group activities.

All sessions focused on the patient-health care professional relationship, with the intent of recognizing how patients are positively affected by a collaborative approach to patient care. Small-group activities were valued less in achieving this goal.

Surveys asked whether students "do not recommend," "neutral/neither recommend nor not recommend," or "highly recommend" each topic/session. All topics had neutral to high agreement ratings (Table 2). Furthermore, students self-reported gaining an appreciation for other professionals' perspectives on the course topics.

Qualitative feedback was provided on course content and overall IPE experiences. Constructive feedback focused on challenges presented due to the time of day or point in the semester that made engagement more difficult (eg, evenings, examinations), or the perceived utility of the lectures or small-group exercises. Overall, each topic had positive ratings on some aspect of IPE reflected on the survey. 
Table 2.

Student Responses to Survey Items by IPE Session ${ }^{\text {a }}$

\begin{tabular}{|c|c|c|c|c|c|c|c|}
\hline Item & Session 1 & Session 2 & Session 3 & Session 4 & Session 5 & Session 6 & Session 7 \\
\hline \multicolumn{8}{|c|}{ The small-group activities were valuable to my IPE and learning. } \\
\hline All students & $88.6 \%(70 / 79)$ & $88.9 \%(64 / 72)$ & $85.19 \%(69 / 81)$ & $81 \%(68 / 84)$ & $69.0 \%(58 / 84)$ & $55.3 \%(42 / 76)$ & $89.3 \%(25 / 28)$ \\
\hline Osteopathic medicine & $60 \%(6 / 10)$ & $69.23 \%(9 / 13)$ & $47.06 \%(8 / 17)$ & $58.8 \%(10 / 17)$ & $40 \%(10 / 25)$ & $52.4 \%(11 / 21)$ & $80 \%(8 / 10)$ \\
\hline Clinical psychology & $87.5 \%(14 / 16)$ & $81.8 \%(9 / 11)$ & $90.9 \%(20 / 22)$ & $75 \%(15 / 20)$ & $75 \%(15 / 20)$ & $47.1 \%(8 / 17)$ & $100 \%(8 / 8)$ \\
\hline Physician assistant & $100 \%(36 / 36)$ & $97.1 \%(33 / 35)$ & $96.4 \%(27 / 28)$ & $92.6 \%(25 / 27)$ & $85.7 \%(24 / 28)$ & $60 \%(15 / 25)$ & $0 \%(0 / 1)$ \\
\hline
\end{tabular}

The course lecture was valuable to my IPE and learning.

\begin{tabular}{|c|c|c|c|c|c|c|c|}
\hline All students & $75.96 \%(60 / 79)$ & $75 \%(54 / 72)$ & $85.19 \%(69 / 81)$ & $65.5 \%(55 / 84)$ & $73.8 \%(62 / 84)$ & NA & $92.8 \%(26 / 28)$ \\
\hline Osteopathic medicine & $50 \%(5 / 10)$ & $61.5 \%(8 / 13)$ & $52.9 \%(9 / 17)$ & $70.6 \%(12 / 17)$ & $52 \%(13 / 25)$ & NA & $80 \%(8 / 10)$ \\
\hline Clinical psychology & $75 \%(12 / 16)$ & $72.73 \%(8 / 11)$ & $86.3 \%(19 / 22)$ & $70 \%(14 / 20)$ & $70 \%(14 / 20)$ & NA & $100 \%(8 / 8)$ \\
\hline $\begin{array}{l}\text { Mental health } \\
\text { counseling }\end{array}$ & $58.82 \%(10 / 17)$ & $76.9 \%(10 / 13)$ & $100 \%(14 / 14)$ & $85 \%(17 / 20)$ & $81.8 \%(9 / 11)$ & NA & $100 \%(9 / 9)$ \\
\hline Physician assistant & $91.7 \%(33 / 36)$ & $80 \%(28 / 35)$ & $96.4 \%(27 / 28)$ & $74.1 \%(20 / 27)$ & $92.9 \%(26 / 28)$ & & $100 \%(1 / 1)$ \\
\hline
\end{tabular}

The small-group activities gave me new ways to think about my role in relation to other health care professionals.

\begin{tabular}{|c|c|c|c|c|c|c|c|}
\hline All students & $82.28 \%(65 / 79)$ & $76.3 \%(55 / 72)$ & $77.78 \%(\mathrm{~N}=81)$ & $76.2 \%(62.84)$ & $69 \%(58 / 84)$ & $64.5 \%(49 / 76)$ & $82 \%(23 / 28)$ \\
\hline Osteopathic medicine & $40 \%(4 / 10)$ & $69.2 \%(9 / 13)$ & $37.1 \%(8 / 17)$ & $47.1 \%(8 / 17)$ & $52 \%(13 / 25)$ & $57.1 \%(12 / 21)$ & $90 \%(9 / 10)$ \\
\hline Clinical psychology & $75 \%(12 / 16)$ & $72.7 \%(8 / 11)$ & $77.2 \%(17 / 22)$ & $75 \%(15 / 20)$ & $58 \%(12 / 20)$ & $58.8 \%(10 / 17)$ & $75 \%(6 / 8)$ \\
\hline $\begin{array}{l}\text { Mental health } \\
\text { counseling }\end{array}$ & $76.5 \%(13 / 17)$ & $77.1 \%(10 / 13)$ & $85.7 \%(12 / 14)$ & $80 \%(16 / 20)$ & $72.7 \%(8 / 11)$ & $69.2 \%(9 / 13)$ & $88.9 \%(8 / 9)$ \\
\hline Physician assistant & $100 \%(36 / 36)$ & $80 \%(28 / 35)$ & $72.86 \%(26 / 28)$ & $92.6 \%(25 / 27)$ & $89.3 \%(25 / 28)$ & $72 \%(18 / 25)$ & $0 \%(0 / 1)$ \\
\hline
\end{tabular}

The course lecture gave me new ways to think about my role in relation to other health care professionals.

\begin{tabular}{|c|c|c|c|c|c|c|c|}
\hline All students & $65.8 \%(52 / 79)$ & $68.5 \%(39 / 72)$ & $74.08 \%(60 / 81)$ & $70.2 \%(59 / 84)$ & $76.2 \%(64 / 84)$ & NA & $82 \%(23 / 28)$ \\
\hline Osteopathic medicine & $50 \%(5 / 10)$ & $46.2 \%(6 / 13)$ & $47.1 \%(8 / 17)$ & $41.2 \%(7 / 17)$ & $56 \%(14 / 25)$ & NA & $70 \%(7 / 10)$ \\
\hline Clinical psychology & $56.3 \%(9 / 16)$ & $63.6 \%(7 / 11)$ & $68.2 \%(15 / 22)$ & $65 \%(13 / 20)$ & $75 \%(15 / 20)$ & NA & $87.5 \%(7 / 8)$ \\
\hline $\begin{array}{l}\text { Mental health } \\
\text { counseling }\end{array}$ & $76.5 \%(13 / 17)$ & $69.2 \%(9 / 13)$ & $78.6 \%(11 / 14)$ & $75 \%(15 / 20)$ & $81.8 \%(9 / 11)$ & NA & $100 \%(9 / 9)$ \\
\hline Physician assistant & $94.4 \%(34 / 36)$ & $77.1 \%(27 / 35)$ & $92.9 \%(26 / 28)$ & $88.9 \%(24 / 27)$ & $92.9 \%(26 / 28)$ & & $0 \%(0 / 1)$ \\
\hline
\end{tabular}

The small-group exercises gave me new ways to think about the professional-patient relationship.

\begin{tabular}{|c|c|c|c|c|c|c|c|}
\hline All students & $78.4 \%(62 / 79)$ & $81.9 \%(59 / 73)$ & $74.08 \%(60 / 81)$ & $70.2 \%(59 / 84)$ & $69 \%(58 / 84)$ & $67.1 \%(51 / 76)$ & $78.6 \%(22 / 28)$ \\
\hline Osteopathic medicine & $50 \%(5 / 10)$ & $69.2 \%(9 / 13)$ & $29.4 \%(5 / 17)$ & $41.2 \%(7 / 17)$ & $48 \%(12 / 25)$ & $76.2 \% 16 / 21)$ & $60 \%(6 / 10)$ \\
\hline Clinical psychology & $75 \%(12 / 16)$ & $63.6 \%(7 / 11)$ & $77.3 \%(17 / 22)$ & $60 \%(12 / 20)$ & $65 \%(13 / 20)$ & $64.7 \%(11 / 17)$ & $87.5 \%(7 / 8)$ \\
\hline $\begin{array}{l}\text { Mental health } \\
\text { counseling }\end{array}$ & $70.6 \%(12 / 17)$ & $76.9 \%(10 / 13)$ & $92.9 \%(13 / 14)$ & $85 \%(17 / 20)$ & $81.8 \%(9 / 11)$ & $61.5 \%(8 / 13)$ & $100 \%(9 / 9)$ \\
\hline Physician assistant & $91.7 \%(33 / 36)$ & $94.3 \%(33 / 35)$ & $89.3 \%(25 / 28)$ & $85.2 \%(23 / 27)$ & $85.7 \%(24 / 28)$ & $64 \%(16 / 25)$ & $0 \%(0 / 1)$ \\
\hline
\end{tabular}




\section{Table 2 (continued).}

Student Responses to Survey Items by IPE Session ${ }^{a}$

\begin{tabular}{|c|c|c|c|c|c|c|c|}
\hline Item & Session 1 & Session 2 & Session 3 & Session 4 & Session 5 & Session 6 & Session 7 \\
\hline \multicolumn{8}{|c|}{ I recommend the course (small group and lecture) be included in the IPE curriculum in future years. } \\
\hline All students & $83.5 \%(66 / 79)$ & $87.5 \%(63 / 72)$ & $90.1 \%(73 / 81)$ & $83 \%(70 / 84)$ & $79.8 \%(67 / 84)$ & $72.4 \%(55 / 76)$ & $89.3 \%(25 / 28)$ \\
\hline Osteopathic medicine & $50 \%(50 / 50)$ & $70 \%(9 / 13)$ & $70.6 \%(12 / 17)$ & $52.9 \%(9 / 17)$ & $60 \%(15 / 25)$ & $76.2 \%(16 / 21)$ & $80 \%(8 / 10)$ \\
\hline Clinical psychology & $87.5 \%(14 / 16)$ & $72.7 \%(8 / 11)$ & $90.9 \%(20 / 22)$ & $85 \%(17 / 20)$ & $85 \%(17 / 20)$ & $70.6 \%(12 / 17)$ & $100 \%(8 / 8)$ \\
\hline Physician assistant & $100 \%(36 / 36)$ & $97.2 \%(34 / 35)$ & $96.4 \%(27 / 28)$ & $100 \%(27 / 27)$ & $89.3 \%(25 / 28)$ & $64 \%(16 / 25)$ & $0 \%(0 / 1)$ \\
\hline \multicolumn{8}{|c|}{ I gained an appreciation for other professionals' perspectives on the topic of the course. } \\
\hline All students & $84.8 \%(67 / 79)$ & $79.1 \%(59 / 72)$ & $83.95 \%(68 / 81)$ & $72.6 \%(61 / 84)$ & $67.9 \%(57 / 84)$ & $68.4 \%(52 / 76)$ & $73.3 \%(21 / 28)$ \\
\hline Osteopathic medicine & $80 \%(8 / 10)$ & $76.9 \%(10 / 13)$ & $41.2 \%(7 / 17)$ & $47 \%(25 / 27)$ & $56 \%(14 / 25)$ & $57.1 \%(12 / 21)$ & $70 \%(7 / 10)$ \\
\hline $\begin{array}{l}\text { Mental health } \\
\text { counseling }\end{array}$ & $70.6 \%(12 / 17)$ & $70 \%(9 / 13)$ & $92.9 \%(13 / 14)$ & $80 \%(16 / 20)$ & $63.6 \%(7 / 11)$ & $61.5 \%(8 / 13)$ & $88.9 \%(8 / 9)$ \\
\hline Physician assistant & $97.2 \%(35 / 36)$ & $85.7 \%(30 / 35)$ & $100 \%(28 / 28)$ & $92.6 \%(25 / 27)$ & $82.1 \%(23 / 28)$ & $80 \%(20 / 25)$ & $0 \%(0 / 1)$ \\
\hline
\end{tabular}

a Session 1, impairment/burnout; session 2, self-care; session 3, elder abuse; session 4, care of intellectually disabled patients; session 5 , business of health care; session 6 , when cultures meet; session 7 , care of physically disabled patients.

Abbreviation: IPE, interprofessional education.

Facilitators' effectiveness was also rated. Students gave positive feedback about the facilitator pairs and their effectiveness. At times, some students from MHC found programming to be medicine-centric, or less relevant to their practice goals.

\section{Discussion}

Interprofessional education requires commitment and collaboration. Despite interested parties and willing participants, the logistics present challenges to skillfully developing IPE curricula. Philadelphia College of Osteopathic Medicine's self-study, planning, and development of IPE over 2 years revealed strengths, weaknesses, opportunities, and challenges that were addressed successfully.

Evaluating content, technology use, small-group activities, and lectures, the IPE course described was successful, according to student surveys. Sessions with direct patient contact, video presentations, and/or roleplaying of patients had the highest value ratings. Use of Google Docs, Google Sheets, and mobile-optimized Blackboard resources made coordination and sharing of materials manageable for students and facilitators and allowed logistics to be handled well.

The strengths of PCOM's IPE included supportive administration and service departments, enthusiastic volunteer faculty, and an existing culture of fostering holistic and collaborative care, in keeping with the tenets of osteopathic medicine (Table 1). Graduate and medical faculty have lectured annually for each others' programs to support this culture. Inviting patients to participate proved valuable in providing practice context. The health services psychology and cognitivebehavioral foci of PCOM's psychology programs have had a biopsychosocial and spiritual approach to patient care since their inception in the early 1990s. Also, because the PCOM health care centers have been 
providing psychology and medicine team-based services for years, IPE was a practiced concept that was easily intellectually supported in the classroom.

Weaknesses or challenges to the development of this IPE course included different cultures of learning and instruction across programs, varied experience and knowledge levels of students, and schedules (DO and PA students attend daytime classes; PsyD and MHC students attend evening classes). Classroom and faculty availability, examination and rotation schedules, and levels of training also required expert problem-solving to coordinate IPE courses. Also, because IPE is a new requirement for most accrediting bodies and institutions, PCOM does not yet have a formal administrative infrastructure or budget for IPE. Thus, faculty from respective departments formed a coalition to help meet the goal to produce competent health care professionals who embrace interprofessional and collaborative teams in health care settings. The work-study program funded PsyD student facilitators' participation; all other clinical and academic faculty were volunteers.

An innovating curriculum with the support of the PCOM administration has allowed for communication about needs and challenges identified within and across disciplines. The bottom-up budgeting process at PCOM allows for and has responded favorably to budget requests with supporting data of time, effort, and necessities for delivering quality education. Furthermore, IPEC hosts webinars and biannual conferences to assist programs in developing IPE, and faculty have been supported in attending these conferences to learn new pedagogy. Given PCOM's outcomes-based culture, psychology team members easily conduct needs assessments, course evaluations, satisfaction surveys, and related dissertation research on the IPE course and aspects of the curriculum, culture, and student body. Interdisciplinary research has been stimulated by the frequent interactions among faculty and increased understanding of areas of expertise.

Challenges to successful IPE engagement appear to be largely external. Students across disciplines have preconceived ideas about health care and roles they expect to assume in delivery of their services. If their prior experiences have led to biases or limited exposure to the culture of interprofessional or team-based, collaborative, integrative care, they may be skeptical about the need for this education. Until external board, licensure, and competency examinations require graduates to have competency in IPEC areas, health profession students may be less inclined to embrace this new learning style. Likewise, until internships, residencies, and postdoctoral training require these prerequisites, there will also likely be some resistance. Without these external requirements, allocating budgetary funds to IPE may be challenging to institutions.

\section{Future Directions}

The 2018-2019 IPE curriculum has been enhanced. Instructional modules are now in block scheduling (eg, 2 introductory sessions on IPE, self-care and burnout; 3 business of health care sessions; and 2 elder abuse sessions), in which students stay with their interprofessional small group longer. Standardized patient exercises are being used to practice the IPEC competencies, to provide work samples for objective evaluation of each student in the collaborative patient encounter, and to form the basis of discussion about IPEC competencies in small groups. Students will begin the process of learning to evaluate peers and give cross-discipline feedback. Student surveys directly inquire about the IPEC competencies with regard to the value of each component of the classes. Thus, the opportunities to learn about, from, and with each other is being maximized. Other IPE research and clinical experiences, training, and curriculum development for advanced students are also underway.

\section{Conclusion}

Interprofessional education requires a universal culture shift external to specific institutions, and PCOM is meeting the demand for IPE and advancing daily. As an institution, PCOM realizes that shifts in mindset at all organizational levels will be critical to sustain 
progress and promote innovation and integration across professional programs.

\section{Acknowledgments}

The following facilitators are acknowledged for their ongoing contribution to the success of the PCOM IPE course: Courtney Ailo, MS; Peter Bidey, DO; Jolene Bohensky, MS, PA-C; Benjamin Chan, DO; Charmaine Chan, DO; David Charney, MS; Jill Cunningham, MHS, PA-C; Nicole Daher, MS, PA-C; Kelsey Dailey, MS; Jay Feldstein, DO; Larry Finkelstein, DO; Nicole Fleischer, MS; Sean Guinane, DHSc, PA-C; Danielle Henderson, PsyD; Kate Laramie, MHS, PA-C; Alexandra Meunch, MS; Michael Serulevich, DO; Wiliam LaValle, PsyD; Anna Zachercenko, PsyD.

\section{References}

1. Health Service Psychology Education Collaborative. Professional psychology in health care services: a blueprint for education and training. Am Psychol. 2013;68(6):411-426. doi:10.1037/a0033265

2. Gilbert JHV, Yan J, Hoffman SJ. A WHO report: framework for action on interprofessional education and collaborative practice. Allied Health. 2010;39 (suppl 1):196-197.

3. Belar $C D$. Reflections on the future: psychology as a health profession. Prof Psychol Res Pract. 2012;43(6):545-550. doi:10.1037/a0028899

4. Schmitt M, Blue A, Aschenbrener CA, Viggiano TR. Core competencies for interprofessional collaborative practice: reforming health care by transforming health professionals' education. Acad Med. 2011;86(11):1351. doi:10.1097/ACM.0b013e3182308e39

5. Brown B, Brehm B, Dodge HS, et al. Evaluation of an interprofessional elective course for health professions students: teaching core competencies for interprofessional collaborative practice. Health Interprof Pract. 2016;3(1). doi:10.7710/2159-1253.1103

6. Kangas S, Rintala TM, Jaatinen P. An integrative systematic review of interprofessional education on diabetes. J Interprof Care. 2018;32 (6):706-718. doi:10.1080/13561820.2018.1500453

7. Welsch LA, Hoch J, Poston RD, Parodi VA, Akpinar-Elci M. Interprofessional education involving didactic TeamSTEPPS $₫$ and interactive healthcare simulation: a systematic review. J Interprof Care. 2018;32(6):657-665. doi:10.1080/13561820.2018.1472069

8. Marcussen M, Nørgaard B, Arnfred S. The effects of interprofessional education in mental health practice: findings from a systematic review. Acad Psychiatry. 2019;43(2):200-208. doi:10.1007/s40596-018-0951-1
9. Sunguya BF, Hinthong W, Jimba M, Yasuoka J. Interprofessional education for whom? - challenges and lessons learned from its implementation in developed countries and their application to developing countries: a systematic review. PLoS ONE. 2014;9(5):1-17. doi:10.1371/journal.pone.0096724

10. Cahn PS. In and out of the curriculum: an historical case study in implementing interprofessional education. J Interprof Care. 2014;28 (2):128-133. doi:10.3109/13561820.2013.872607

11. Tartavoulle TM, English R, Gunaldo TPet al., Using the IDEA framework in an interprofessional didactic elective course to facilitate positive changes in the roles and responsibility competency. $J$ Interprof Educ Pract. 2016;2:21-24. doi:10.1016/j.xjep.2016.03.003

12. Frantz JM, Rhoda AJ. Implementing interprofessional education and practice: lessons from a resource-constrained university. J Interprof Care. 2017;31(2):180-183. doi:10.1080/13561820.2016.1261097

13. O'Keefe M, Ward H. Implementing interprofessional learning curriculum: How problems might also be answers. BMC Med Educ. 2018;18(1):132. doi:10.1186/s12909-018-1231-1

14. Brashers V, Peterson C, Tullmann D, Schmitt M. The University of Virginia interprofessional education initiative: an approach to integrating competencies into medical and nursing education. J Interprof Care. 2012;26(1):73-75. doi:10.3109/ 13561820.2011 .636846

15. Tenets of osteopathic medicine. American Osteopathic Association website. https://osteopathic.org/about/leadership/aoa-governancedocuments/tenets-of-osteopathic-medicine/. Accessed August 5, 2019

16. Oshry B. When cultures meet workshop. Power + systems website. https://www.powerandsystems.com/workshops-certifications/ merging-cultures.html. Accessed August 4, 2019

17. Carman M. Training and Development: The Power of Simulation-Based Learning. Sydney, Australia: Michael Carman Consulting; 2013.

18. Reid M, Brown S, Tabibzadeh K. Capstone teaching models: combining simulation analytical intuitive learning processes, history and effectiveness. J Educ Business. 2012;87(3):178-184. doi:10.1080/ 08832323.2011 .586006

19. Kim H, MacDonald RH, Andersen DF. Simulation and managerial decision making: a double-loop learning framework. Public Adm Rev. 2013;73(2):291-300. doi:10.1111/j.1540-6210.2012.02656.x

20. Swain GR, Grande KM, Hood CM, Inzeo PT. Health care professionals: opportunities to address social determinants of health. WMJ. 2014;113(6):218-222

C) 2019 American Osteopathic Association 